\title{
Research Paper: The Effect of Lower Extremity Mus- cle Fatigue on Dynamic Postural Control Analyzed by Electromyography
}

\author{
Majid Fatahi $^{{ }^{*}}$, Gholam Ali Ghasemi², Yosef Mongashti Joni ${ }^{3}$, Vahid Zolaktaf ${ }^{2}$, Faraj Fatahi ${ }^{1}$ \\ 1. Department of Sport Injuries and Corrective Exercises, Faculty of Physical Education \& Sport Sciences, University of Isfahan, Isfahan, Iran \\ 2. Department of Pathology and Corrective Exercises, Faculty of Physical Education \& Sport Sciences, University of Isfahan, Isfahan, Iran. \\ 3. Department of Management and Sport Sciences, Faculty of Physical Education \& Sport Sciences, Urmia University, Urmia, Iran.
}

Citation: Fatahi M, Ghasemi GHA, Mongashti Joni Y, ZolaktafV, Fatahi F. The Effect of Lower Extremity Muscle Fatigue on Dynamic Postural Control Analyzed by Electromyography. Physical Treatments. 2016; 6(1):37-50. https://doi.org/10.18869/NRIP.PTJ.6.1.37

do: : https://doi.org/10.18869/NRIP.PTJ.6.1.37

Article info:

Received: 29 Sep. 2015

Accepted: 10 Jan. 2016
Keywords:

Electromyography, Postural control, Fatigue, Y balance, Lower extremity muscles

\section{A B S T RA C T}

Purpose: Postural control preserves organs and body parts in a proper biomechanical stance which exists in two forms: static and dynamic. Fatigue is one of the factors that affects postural control. This study aimed to compare the electromyography (EMG) activity of the lower extremity muscles before and after fatigue.

Methods: This study was descriptive correlational and based on the research type a field study. Study sample comprised 20 male students majored in physical education; they were purposefully selected by non-accidental all-accounted method. Surface EMG activities of lower extremity muscles before and after fatigue were evaluated by electromyogram. To create fatigue in lower extremity muscles, we used fatigue protocol by the Biodex system. Data analysis was carried out by using SPSS 21. The paired t test was used for statistical analysis with a significance level of $\mathrm{P}<0.05$.

Results: The paired $t$ test results indicated that the activity level of the rectus femoris, hamstrings, tibialis anterior, and gastrocnemius muscles significantly changed before and after fatigue. The study results also supported that lower extremity muscle fatigue had a negative effect on the activity of the muscles around the knee joint. Furthermore, there was a significant relationship between the postural control and the activity level of rectus femoris and tibialis anterior muscles on pretest. However, there were no significant relationships between postural control and activity level of lateral hamstrings and gastrocnemius muscles on pretest and posttest, nor the activity level of rectus femoris and tibialis anterior posttest.

Conclusion: Muscle fatigue increases joints vulnerability. These results can be used in designing athlete's rehabilitation programs and trainings to prevent injuries or changes in biomechanical parameters of walking.

\section{*Corresponding Author:}

Majid Fatahi, $M S c$.

Address: Department of Sport Injuries and Corrective Exercises, Faculty of Physical Education \& Sport Sciences, University of Isfahan, Isfahan, Iran .

Phone: +98 (916) 3056603

E-mail:fatahi879913@yahii.com 


\section{Introduction}

$\mathbf{P}$

ostural control is the key factor and essential for daily activities and exercises. It maintains the organs and different body parts in an appropriate biomechanical stance which exists in the two forms of static and dynamic [27]. Postural control is the quantitative measurement of neuromuscular control that plays an important role in the dynamic joint stability and keeping the body away from the injuries [38]. Postural control is achieved through biomechanical and sensorimotor processes. It results from contribution of three systems; visual, vestibular, and somatosensory. The cooperation of these systems leads to postural control and balance [32].

The research indicates that the muscles of the lower extremity have a very important role in maintaining postural control, especially in single-leg movements. Inefficiency or weakness of these muscles during dynamic movements may disturbs the dynamic postural control, affects the stability of the posture, and leads to imperfect movements. Performing daily activities and exercises considerably depends on keeping balance which is defined as the ability to maintain or return the body center of mass within the support base or control and keep the body position in space to achieve stability and orientation. Balance is the most important factor in doing exercise [15]. It is a complicated skill which describes the dynamic posture of the body in preventing from falling [13]. Various factors affect the person's ability in keeping and recovering the postural control of the body; the most important of them are nervous system injuries, inefficiency of the optic nerves, stress, vestibular system defects, and fatigue. To study the effects of these factors on the body control system, special laboratory, equipment, and field tools are used [17].

Muscular fatigue decreases the performance of the metabolic and neuromuscular systems which results in persistent muscle contraction and reduction in its steady activity. Thus, muscle contraction cannot continue for a long time [3]. This is an unpleasant phenomenon which may happen after a short- or long-term activity (maximal or submaximal) [4]. Research indicates that muscle fatigue increases postural sway and reduces the ability to keep the balance [16]. Fatigue is a transient disability in keeping the muscle strength to perform consecutive contractions [25].

With respect to the nervous system, fatigue is categorized into central and peripheral types. Lack of activation and sending impulses from central nervous system to the muscles and their inability for producing proper tension and response are defined, respectively, as the main causes of the central and peripheral fatigue [1] Because most exercises are done in a dynamic condition, dynamic balance is one of the most important factors of fitness and doing sport activities [10].

Gribble and Hertel (2004) demonstrated that thigh muscle fatigue can impair postural control in sagittal and frontal planes and affect body functions [14]. Di Giulio et al. (2009) reported a high correlation between EMG activity of the tibialis anterior muscle and body sway (as a function) in the standing position. These results indicated a relationship between the EMG activity of the lower extremity muscles and displacement of the body center of the gravity. They have also reported a moderate relationship between the EMG activities of the gastrocnemius and soleus muscles with body sway in standing position [9].

Most sport injuries occur near the end of the sport activities and competitions. Thus, the negative and accumulative effects of fatigue, especially near the end of the competitions, on the neuromuscular control can lead to dangerous movement strategies and an increase in the risk of injury. The results indicated that fatigue not only decreases the muscle capacity in producing energy but also deeply affects the coordination of movements, motor control acuity, muscle reaction time, and proprioception ability, which causes a significant reduction in muscle performance [24]. This negative effect is important with regard to injury prevention because muscles, besides contraction, have other duties, such as reduction of impact forces, bending forces on bones, and increasing joint stability. If muscles perform their functions well, they can support body against injuries. Any changes in muscle function due to fatigue reduces muscle ability to prevent sport injuries [36].

Today, the electromyography (EMG) is widely used in all areas of medicine, rehabilitation, and sport. EMG is an experimental technique in which the electrical signals of the muscle are detected, recorded, and analyzed. It has several advantages which "monitoring the electrical activity of the muscle directly and without intermediaries" might be its most important advantage [22]. This study aimed to examine the relationship between lower extremity muscle activity with using tests of lower limb performance and postural control and also the effect of fatigue by using EMG. In this study, the Y Balance Test was used to assess the dynamic postural control. This test is a valid and reliable instrument to quantify the dynamic postural control [25].

\section{Materials and Methods}

This research is a descriptive correlational and field study. The research design is intragroup with pretest 
and posttest of the study variables; EMG activity of the lower limb muscles and postural control before fatigue and after administering fatigue protocol.

\section{Study participants}

The present study was conducted on the male students of the Faculty of Physical Education and Sport Sciences University of Isfahan who enrolled in the academic year 2013-2014. The study subjects were 20 male students (mean (SD) age: 24.9(2.44) y, mean (SD) weight: $69.16(7.51) \mathrm{kg}$, mean (SD) height: $175.05(4.54) \mathrm{cm})$ who were chosen purposefully. Subjects with a history of neuromuscular disorders, fracture or surgery of the lower limbs during the past six months, ankle sprains in the past year, lower limb abnormalities such as valgus deformity, flat foot and arch foot, and vestibular impairment were excluded from the study. All tests were performed in the laboratory of Isfahan University, Faculty of Physical Education. Before the study, the experimental protocol was explained to all participants who all signed informed consent forms.

\section{Study procedure}

The Y Balance Test was used to measure the dynamic balance of the participants. This is a test based on the star excursion balance test (SEBT) which Grybl et al. (2012) considered it as a valid test for the evaluation of dynamic balance [5]. SEBT is a reliable and validated test to predict lower extremity injuries, determine the dynamic balance impairments in people with lower extremity injuries, ascertain the effects of fatigue on the balance, and also the effects of exercise to improve balance.

A

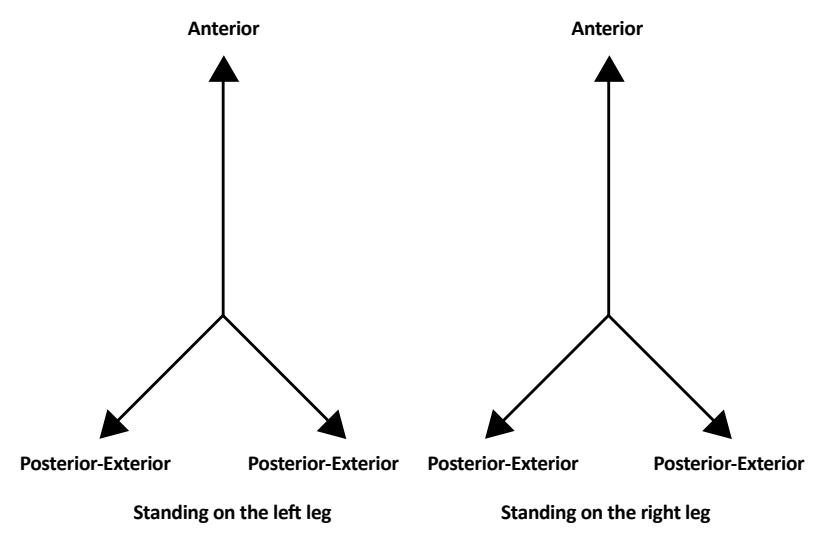

Figure 1. $Y$ Balance Test.
Taking this test needs power, flexibility, neuromuscular control, core stability, proprioception, and balance; therefore, it is an excellent-test for evaluating pre-season and medical assessments [5]. Plisky et al. (2009) designed the Y Balance Test to evaluate dynamic balance and to standardize SEBT assessment [35]. In this test, three directions (anterior, posteromedial, and posterolateral) are drawn at an angle of $135^{\circ}$ to each other. Because of significant correlation of this test with leg length, to perform this test and normalize the data, the leg length was measured from the anterior superior iliac spine to the medial malleolus in the supine position while the subject lying on the ground [6].

To run the test, each subject with one leg (the dominant one) practiced the test 4 times to learn test procedure (the subject with right dominant leg performed the test in the counterclockwise direction, and the subject with the left dominant leg did it in the clockwise direction). The subject stood at the center of the test on one leg and with the other leg, in the direction which the examiner chose, performed the error-free maximum-reach performance and then returned to the normal position. To eliminate the effect of learning, each subject practiced each direction, 4 times with 15-second intervals rest. After a 5-minute break, the subject started the test in the direction that the examiner had chosen randomly (Figure 1).

The examiner measured the subject's leg location to the center of the test in centimeter. The test was repeated 3 times for the subjects. The best record was divided by the leg length, and then multiplied by 100 until the reach distance in terms of leg length was obtained. If an error occurred during the test such as subject's stance
B

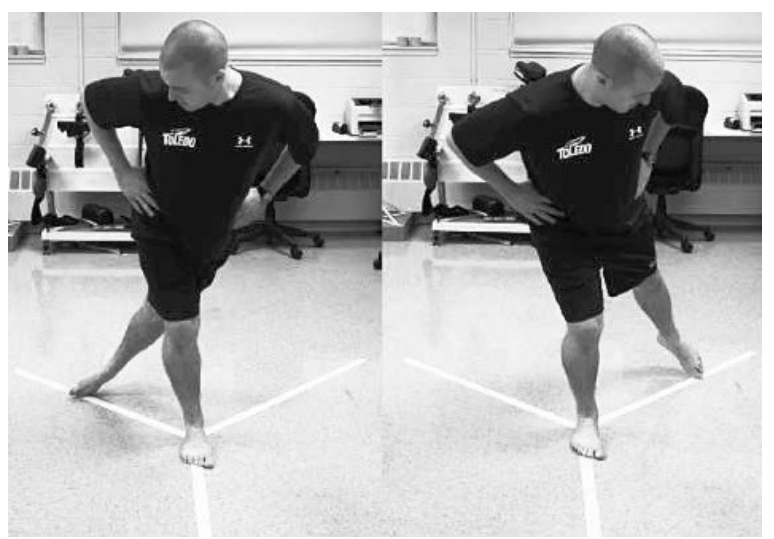

A: Schematic view of $Y$ Balance Test

$\mathrm{B}$ : The subject performing the Y Balance Test. 
leg moved out the center or his balance was disrupted, the subject would be asked to repeat the test [35].

\section{Data collection}

The measurement procedure can be divided into 5 steps:

- Filling out the questionnaire and warming up;

- Preparing subjects and electrode placement;

- Measuring lower extremity EMG activity by using tests of lower extremity function under normal conditions (without exhaustion);

- Administering lower extremity muscle fatigue protocol by using Biodex system; and

- Measuring lower extremity EMG activity by using lower extremity function test after the fatigue protocol.

\section{Filling out the questionnaire and warming up}

At the beginning of each session, the researcher explained to each subject the purpose of the research, its measuring instruments, and the process. After providing this information and subject's willingness to participate in this study, a questionnaire was handed to the subject to provide the required information. After filling out the questionnaire, he was asked to participate in the test with sport clothes. Participants' leg length and height were measured and recorded by the tape and a digital scale, respectively. The dominant leg of the subject was determined by the examiner. Next, the researcher taught the participants how to do the lower extremity function in the test.

\section{Preparing samples and electrode placement}

Since much information about the range of EMG variables in this study was related to lower limb muscles, subjects' preparation and installation of surface electrodes were conducted in the following way. First, according to the instructions provided by the examiner, each subjects shaved all the skin appendages, coarse and fine hairs over electrode placement areas on the quadriceps, hamstring, tibialis anterior, and gastrocnemius muscles by using a disposable personal razor blades.

To reduce ohmic resistance of the skin surface, a special, gentle, and soft sandpaper was used to scrub the skin. Then, by using 5\% isopropyl alcohol, the scrub residuals and skin sweating were wiped out to provide a proper condition to place the electrode pads on the skin surface. Next, the electrode pad placement on the muscles was determined according to SENIAM European Protocol [39].

After specifying the desired muscles, the places of the electrode positions were determined as follows [18]:

- Rectus femoris muscle: In the middle of the line between the anterior superior iliac spine and upper part of patella;

- Lateral hamstring muscle: In the middle of the line between the ischial tuberosity and the lateral epicondyle of tibia;

- Tibialis anterior muscle: Upper third on the line between tip of fibula and ankle; and

- Medial gastrocnemius muscle: The most protruding part.

In addition, to ensure the location of the muscle, the isometric muscle contraction proposed by the Kendall was used to determine the bulk muscle in all cases [44] After determination of the electrode pad placements, they were put and attached on the specified places. Lower extremity EMG activities were measured by using tests of lower extremity function under normal conditions (without exhaustion).

\section{Performing the experiment to record the EMG activity}

To record the EMG activity of the lower extremity, the function tests included the Y Balance Test. At first, the test method was explained to each subject and asked him to perform the test with his maximum power and speed, upon hearing the command of "ready, go" (which was simultaneous with the start of electromyogram). During the test, the subject stood on one leg at the center of the test and moved the other leg in the direction chosen by the experimenter to achieve maximum error-free reach and then returned to normal condition. If an error occurred during the test such as subject's stance leg moved out the center or his balance was disrupted, he would be asked to repeat the test [37]. For Y Balance Test, the distance was calculated by a tape meter; the examiner permitted the start move by holding the EMG instrument.

After finishing the test, the memory card from the surface electromyogram was transferred to the com- 


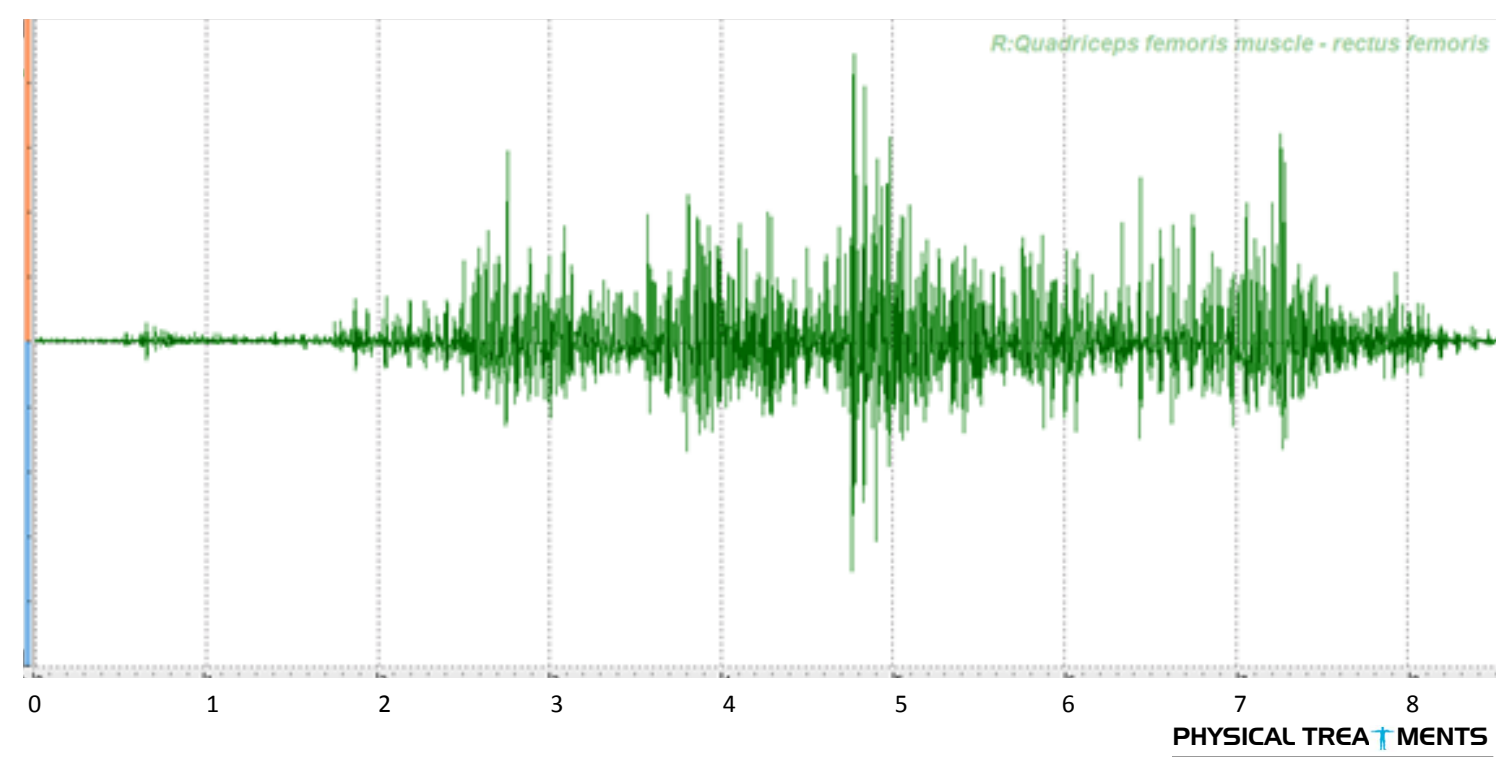

Figure 2. A view of the EMG diagram recorded during the execution of the Y Balance Test.

puter and the recorded EMG waves were analyzed. The electrodes were not detached from the subject's body to repeat the test in case of any mistake found in recording the waves. After being ensured of the correctness of the EMG waves recording, the electrodes were separated from the subject's body and their places were cleaned with water and alcohol.

\section{Lower extremity muscle fatigue protocol}

Among the various methods of evaluating and creating fatigue, voluntary contraction, has been always the first choice and criterion to quantify the fatigue. Furthermore, using the maximum voluntary contraction has been considered as a gold standard [42]. In the current study, administering fatigue protocol to reach $50 \%$ of maximum torque repeated contractions, provides the study feedback, and moreover, it is a reproducible and standardized measure. However, the administration of the motioned fatigue protocol in some similar and previous studies has caused significant changes in balance control indices $[18,38]$.

To administer fatigue in the targeted muscle groups, the Biodex system was used. For this purpose, first the maximum torque of knee flexor and extensor muscles was recorded. To administer fatigue in knee area, the angle of the trunk and knees flexion were set at $110^{\circ}$ and $90^{\circ}$, respectively (at the start). To avoid involving other muscles in the test, subject's trunk, hip, and knees were fixed to the Biodex system by seat belt.

At the beginning of the program, to warm up muscles and familiarize subjects with the movements in the sys- tem, some submaximal movements were performed. Then to record the maximum torque of each subject, three knee flexion and extension moves (to record for maximum muscle torque knee) were carried out with maximum effort and the average of three movements was recorded as the maximum torque. During the study on knee muscles area, the selected contraction was isokinetic with concentric/concentric type. Also, the contraction speed for extension and flexion movements of knee was $360^{\circ}$ per second. It was assumed that when subject's torque after three consecutive moves reduced to less than $50 \%$ of maximum torque (recorded during the first movement), then he would experience fatigue [18]. Lower extremity EMG activities were measured using lower extremity function test after administering the fatigue protocol (Figure 2).

\section{Final processing of EMG data}

Because 4 electrodes were connected to target muscles of each subject, we received 4 raw EMG wave for each subject. To analyze raw EMG waves, RMS computing method was used. Di Fabio algorithm was used to show the muscle activity during functional tests as well as starting and finishing moves of RMS EMG data [8]. On the rest part of the algorithm, a $t_{1}$ point was randomly selected to mark the onset of contraction. Time $t_{2}$ was set 3 to $5 \mathrm{~ms}$ after the time $\mathrm{t}_{1}$. Then, mean RMS between $t_{1}$ and $t_{2}$ was calculated and recorded as the start time. Also, to measure the end of contraction time, on the rest part at the end of the algorithm, a $t_{3}$ point was randomly selected, then $\mathrm{t}_{4}$ time was set 3 to $5 \mathrm{~ms}$ after the time $t_{3}$. Finally, the average RMS was recorded as 


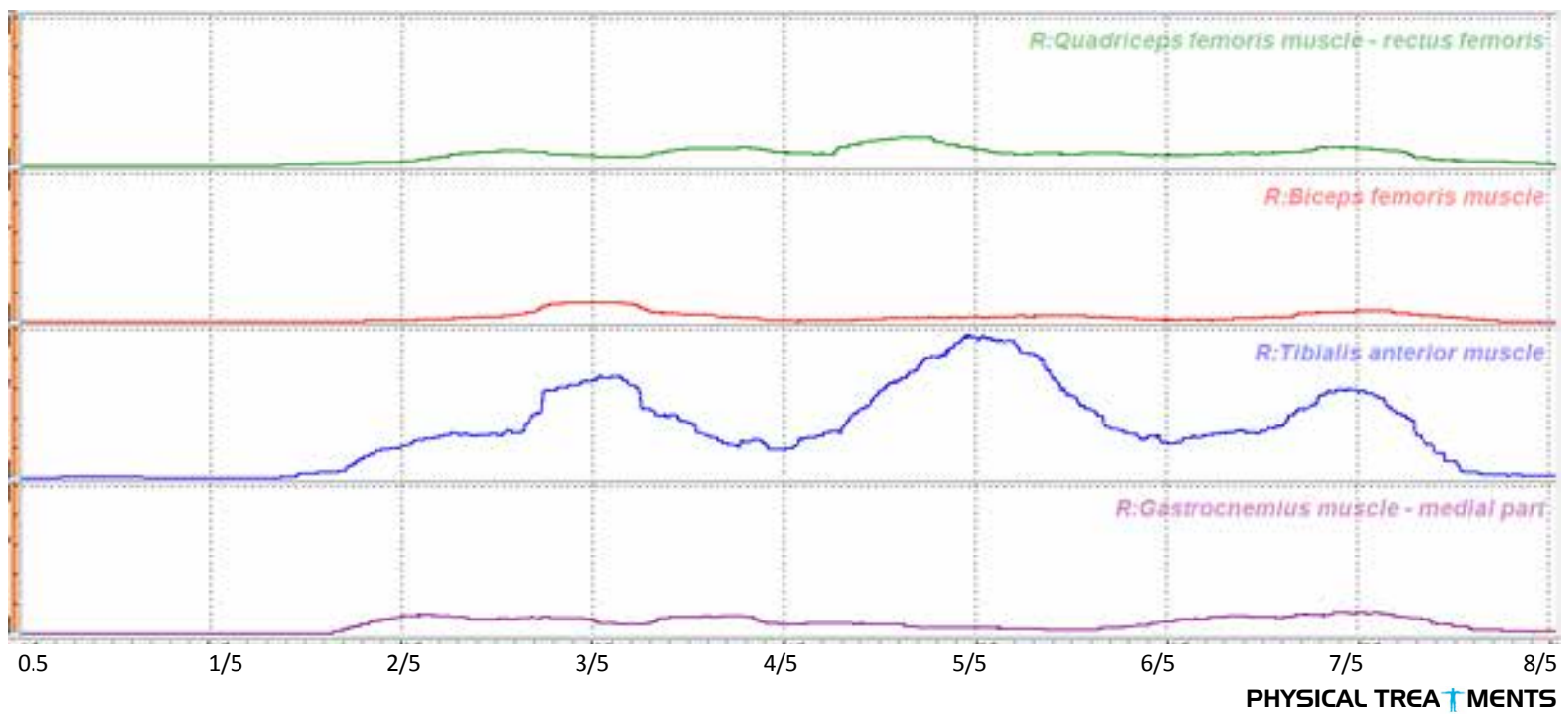

Figure 3. A view of the RMS EMG diagram recorded during the execution of Y Balance Test.

the end of the contraction. In addition, to normalize the data of the start and finishing time, the formula

$$
T h=E M G_{(\text {rest })}+3 S D \text {, }
$$

was used. Next, the mean RMS between the beginning and end of each sample's contraction was used as the raw score for each subject [41].

Raw EMG signals were entered in MegaWin 3.0.1 to calculate the level of muscle activity. With regard to the nature of the RMS signal, there was no need to unidirectionalize the signals first. RMS was also calculated from maximum voluntary isometric contraction data recorded by the Biodex system. For this purpose, RMS was also calculated from raw EMG signal data, which were recorded by the Biodex system. Then, by dividing the amount of obtained activity for each muscle on the MVC and multiplying the resulting number by 100 , the percentage of each muscle activity was determined (Figure 3) [2].

Each isometric maximal voluntary contraction was divided on the RMS data to be normalized and presented. Such analyses were performed on all EMG data obtained from all subjects.

\section{Statistical methods}

After data collection, the subjects' characteristics, including age, height, and weight were analyzed with descriptive and inferential statistics using SPSS 21. Excel 2013 was used to draw the study charts and the graphs. The Kolmogorov-Smirnov test was used to be ensured of the data normality. Also, paired t-test was used to examine the mean difference of the functional tests re- sults before and after the fatigue. Finally, the Pearson correlation coefficient was also used to examine the relationship between the activity of lower limb muscles and postural control before and after the fatigue. All data analyses were done at confidence level of $95 \%$.

\section{Results}

Demographic characteristics of study subjects, including their mean and standard deviation of height, weight, and age are presented in Table 1. Descriptive information (mean and standard deviation) of the measured variables in the study group before and after the fatigue are presented in Table 2. To investigate the changes in each variable, the results of analysis for repeated data are provided in Table 3 .

\section{Features of the variables}

In this study, the level of postural control is the predictor variable measured by function tests. Also the level of EMG activities of the lower limb muscles (quadriceps, biceps femoris, tibialis anterior, and gastrocnemius) were the criteria variables assessed before and after fatigue. The features of these variables are presented in the following tables. To investigate the difference between the average level of the lower limb muscle activities of the subjects before and after the fatigue, the paired t-test was used. The results of this test are presented in Table 4.

Paired t-test results indicated a significant difference between the activity level of rectus femoris muscles, hamstrings, tibialis anterior, and medial gastrocnemius ( $\mathrm{P}<0.01, \mathrm{P}<0.01, \mathrm{P}<0.01, \mathrm{P}=0.02$, respectively) before and after the test. 
Table 1. The study participants' characteristics including age, height, and weight.

\begin{tabular}{ccc}
\hline Subjects' Characteristics & Mean & SD \\
\hline Weight (kg) & 69.16 & 6.99 \\
Height (cm) & 175.05 & 3.74 \\
Age (y) & 24.11 & 1.88 \\
\hline
\end{tabular}

Table 2. The mean and SD of Y Balance Test results, before and after fatigue.

\begin{tabular}{ccccc} 
& & \multicolumn{2}{c}{ After Fatigue } \\
\hline & Mean & SD & Mean & SD \\
\hline $\mathrm{Y}, \mathrm{cm}$ & 80.85 & 8.76 & 76.85 & 10.40 \\
\hline
\end{tabular}

PHYSICAL TREA T MENTS

Table 3. The mean and SD level of the muscles' activities before and after fatigue $(\mathrm{mV})$ during Y Balance Test.

\begin{tabular}{ccccc}
\hline & & \multicolumn{3}{c}{ Y Test Results } \\
\cline { 2 - 5 } & & Before Fatigue & \multicolumn{2}{c}{ After Fatigue } \\
\hline EMG Activity of Muscles & Mean & SD & Mean & SD \\
\hline Rectus femoris & 0.507 & 0.116 & 0.427 & 0.120 \\
\hline Lateral hamstring & 0.525 & 0.086 & 0.404 & 0.141 \\
\hline Anterior tibialis & 0.530 & 0.095 & 0.415 & 0.076 \\
\hline Medial gasterocnemius & 0.600 & 0.093 & 0.438 & 0.119 \\
\hline
\end{tabular}

PHYSICAL TREA TMENTS

The results of the correlation coefficient indicated significant relationships between the rectus femoris and tibialis anterior muscle activity levels with subjects' postural control before the fatigue stage $(\mathrm{P}=0.02, \mathrm{P}=0.04$, respectively). However, there were no significant relationships between the activity level of lateral hamstring and medial gastrocnemius muscles and subjects' postural control before the fatigue $(\mathrm{P}=0.34, \mathrm{P}=0.48$, respectively). Also, there were no significant relationships between the rectus femoris, hamstring, tibialis anterior, and medial gastrocnemius muscles with the subjects' postural control after fatigue ( $\mathrm{P}=0.94, \mathrm{P}=0.61, \mathrm{P}=0.35, \mathrm{P}=0.09$, respectively) (Table 5).

Table 4. Paired t-test results of lower extremity muscles before and after fatigue in the Y Balance Test.

\begin{tabular}{rccc}
\hline & $\mathbf{t}$ & SD & Sig. \\
\hline Rectus femoris & 2.61 & 0.11 & 0.02 \\
\hline Lateral hamstring & 1.66 & 0.18 & 0.00 \\
\hline Tibialis anterior & 4.31 & 0.08 & 0.00 \\
\hline Medial gastrocnemius & 4.07 & 0.14 & 0.00 \\
\hline
\end{tabular}


Table 5: The Pearson correlation coefficient examining the relationship between the postural control and lower limb muscles activities before and after fatigue.

\begin{tabular}{rllll}
\hline & $\mathbf{R}$ & $\mathbf{P}$ & $\mathbf{R}$ & $\mathbf{P}$ \\
\hline Rectus femoris & 0.61 & 0.02 & 0.02 & 0.94 \\
\hline Lateral hamstring & 0.20 & 0.48 & 0.14 & 0.61 \\
\hline Tibialis anterior & 0.64 & 0.04 & 0.33 & 0.35 \\
\hline Medial gastrocnemius & 0.27 & 0.34 & 0.46 & 0.09 \\
\hline
\end{tabular}

PHYSICAL TREA TMENTS

\section{Discussion}

The study results indicated that after lower extremity muscle fatigue protocol, the electrical activity of muscles and the Y Balance Test scores significantly reduced in all directions. Therefore, lower extremity muscle fatigue had a negative effect on dynamic balance of study participants.

Lower extremity besides being the body base and support, moves the body as well. Therefore, the muscles in this part aside from providing stability of the lower extremity in the standing position, affect the body movements in dynamic conditions [30]. The muscles of the lower extremity help pelvic stability and kinetic chain during functional activities. When these muscles work well, they properly generate and distribute the maximum power with minimal compression and shear forces in kinetic chain joints. They also provide optimal control of the movements and absorb properly impact forces during landing due to ground reaction forces [23].

The dynamic balance decline caused by fatigue of lower extremity muscles can be justifiable. Fatigue can decrease the ability in generating forces, neuromuscular coordination, the exact kinetic control, proprioception, muscles stability, and co-contraction of the muscles. It can also increase the reaction time which its main result would be reduction in the muscles performance [31]. Gribble et al. (2004) considered some factors such as power, flexibility, neuromuscular control, core stability, proprioception, and joint range of motion, effective in successful execution of SEBT. They considered this test as a proper test to analyze the effects of the fatigue on the dynamic balance. Considering the negative effects of the fatigue on the muscles which are major players in performing the SEBT, the reduction in the test results seems reasonable [9].

However in this study, the Y Balance Test (which is based on SEBT) was used. To perform the Y Bal- ance Test, the subject, instead of 8 directions in SEBT, performs only three directions of the anterior, posteromedial, and posterolateral. The Y Balance Test is conducted in closed kinetic chain and thigh muscles play an important role in the function and lower extremity alignment during closed kinetic chain activities [42].

Studies have shown that thigh muscles are extremely influential to maintain balance [26]. Hart et al. (2006) observed a reduction in the function of quadriceps muscles after fatigue in trunk extensor muscles of healthy people and sick ones suffering from backache [23, 24]. On the other hand, the results of an EMG study showed the quadriceps muscles are active during all directions of SEBT [10]. Therefore, the reduction in the balance test scores can be attributed to the muscle tiredness due to the fatigue protocol of the lower extremity muscles.

Quadriceps muscles are very important in generating proper torque and controlling knee joint [7]. During standing on one leg, the quadriceps muscles are contracted in an eccentric way to control the lowered center of the body and at the same time reduce the imported loading contacts [26]. Gribble $(2004,2009)$ reported that the changes in the proximal muscle activities due to the fatigue could reduce the range of knee and hip joints motion and finally reduce the reach performance in SEBT $[19,20]$.

Filipa et al. (2009) also believed that muscle coordination and proprioception on performing the SEBT and loss of neuromuscular coordination of the body due to fatigue can negatively affect the stability of lower extremity muscles [15]. When a person tries to maintain the posture, the corrective contractions happen constantly in response to small joints disturbances. Since fatigue can decrease the nerve conduction velocity, the ability to make contractions around the muscles may be reduced which results in the neuromuscular control weakness and more changes around joints. The further 
changes in joints range of motion may reduce the balance in the absence of corrective actions of the muscles.

Our study results indicated that after administration of fatigue protocol, the activity of the rectus femoris muscle reduced significantly. The quadriceps muscles with their concentric contractions control the knee flexion and are ready to open knee and support the body weight. Low muscle activity leads to less torque and impaired control of knee flexion, as well as support of the body weight. Therefore, it may cause problems in body posture. Di Giulio et al. (2009) reported a strong relationship between the EMG activities of tibialis anterior muscle and postural sway (as a function) in standing position. These results indicated a relationship between the EMG activity of the lower extremity and displacement of the body center of gravity.

According to what was said, reduction in the tibialis anterior muscle activities, after administration of the fatigue program, can impair the sensory receptors and balance corrections in this area. Therefore, applying the fatigue protocol can be more influential in postponing balance corrections and as a result, obtaining a shorter reach distance during the $\mathrm{Y}$ Balance Test compared to that distance before fatigue. Thus, the lower extremity muscle activity can reduce the reach distance and weaken dynamic balance because the subject must rely on neuromuscular control around the muscles of lower extremity for reaching longer distances which in the case of untimed start of the balance corrections and lack of generating enough forces, would cause problems for muscles around joints in performing the optimum movement.

According to the study findings, to maintain an optimum dynamic balance during sport activities, the proper level of the muscle activities around lower extremity joints, especially in the anterior region of the leg is very important.

Our study results indicated that the level of the activities in hamstring muscle decreased. Fatigue of the quadriceps muscles may probably affect the co-contraction pattern of the hamstring muscle [12], and thus reduces hamstring muscle activities. Similarly, Parijat (2008) reported the reduction in the hamstring muscle activities during heel contact phase, but he attributed the hamstring muscle activity reduction in walking phase to increase in the speed of heel contact after local fatigue in the quadriceps muscles [42].

Base on the study results, the EMG activities of all studied muscles decreased. The study results were consistent with some other studies like Pincivero et al. (2006) [44]. The reason for a significant difference between the levels of the activity in the lower extremity muscles, before and after fatigue, may be attributed to the fatigue that causes problems in the sensory receptors and balance corrections. Therefore, applying the fatigue program can have a more significant role in postponing the balance corrections and as a result, reaching a shorter distance during the $\mathrm{Y}$ Balance Test compared to that distance before the fatigue.

Also, with regard to more obvious effects of administering fatigue protocol by the Biodex system, one can point to the received massages to the brain from sensory receptors of all parts of body due to the exhaustion. Because applying the fatigue program to the exhaustion stage sends messages from sensory receptors of nearly all muscles to the central nervous system to reduce activities and prevent any injuries.

Therefore, to increase the dynamic balance and decrease the effects of fatigue, and consequently, the probable injuries after physical activities, it is suggested that trainers pay special attention to muscular endurance exercises in planning sport programs and physical fitness, especially in the lower extremities and their anterior part. In summary, fatigue in the lower extremity muscles of the body, probably through a negative effect on neuromuscular coordination, motion control acuity, stability of the proximal joints, and the transfer of such destructive effect to the distal joints, causes functional impairment in chain kinetics [26]. Finally, poor balance decreases the ability in performing skilled activities and limits the functional movements [39].

Also, other findings of this research indicated a significant relationship between the activity levels of the rectus femoris and tibialis anterior muscles with subjects' postural control before fatigue, but there was no such significant relationship after fatigue. Earl and Hertel research indicated that in all directions of SEBT, there were coordinated contractions of the quadriceps and hamstring muscles. Rectus femoris muscle was active during three excursions of the test. To do anterior excursion, the subject lean back and the trunk extension helps maintaining balance. The gravity exerted to the upper body leads to a great momentarily flexion in the knee which must be controlled by the momentarily extension generated by the quadriceps muscles [39].

The study results indicated high activities of the rectus femoris muscle during three directions of the $\mathrm{Y}$ Balance Test which had a significant relationship with reach level in different directions of the test. That is be- 
cause during performing the test, the subject must rely on the neuromuscular control around lower extremity muscles to reach for the maximum distance. To this end, the person must lean backward and the trunk be in the extension position to keep the balance. In this position, the force of gravity acting on the upper part of the body causes a high torque of knee flexion that must be controlled by the extension torque generated by eccentric contraction of quadriceps muscles.

The study results indicated that after applying the fatigue protocol, the activities of quadriceps muscles significantly reduced. Quadriceps muscles of the thigh with a concentric contractions control the knee flexion and are ready to open the knee and support of the body weight $[58,59]$. The reduction in the muscles activities lowers generated torque, disturbs knee flexion control and body support [42], and consequently may lead to problems in the postural control.

By all accounts, decline in the tibialis anterior muscle activity after applying fatigue, will impair the sensory receptors and balance corrections in this area. Low activity of lower extremity muscles will result in decreasing reach distance and dynamic balance, as during the reach process, the subject must rely on the neuromuscular control around lower extremity muscles to reach the maximum distance and without balance correction at the start point and lack of generating enough forces around muscles, there will be problems in performing the proper mentioned movements. Based on the study findings, for maintaining an optimum dynamic balance during sport activities, the level of the proper activities of the performing muscles around lower extremity joints especially in the anterior region of the leg is of most importance.

According to the above mentioned topics, the researcher assumed that if the lower extremity muscles get tired and weak, and lower extremity functional tests are undertaken at this stage, a significant relationship may be found between the level of the muscles activities and postural control. However, the research results indicated that the mentioned relationship was not significant at the time of fatigue. At the present study, the relationship between the muscles activities and postural control differed after fatigue compared to normal condition before fatigue.

In most cases, there was a stronger relationship between postural control and the rectus femoris and tibialis anterior muscle before fatigue, but this relationship was not significant between postural control and activities of the lateral hamstring and medial gastrocnemius muscles. In other words, it did not follow any specific and fixed pattern to deduce a correlation. With regard to previous discussions, because the Y Balance Test is conducted in a closed kinetic chain, the produced changes resulted from fatigue in proximal parts affect the distal parts and reduce the Y Balance Test scores. Therefore, the function of the lower extremity muscles influences the dynamic balance of male university athletes in this study.

Postural control is the key factor for performing the daily activities and exercises. With regard to the effects of strength, range of motion, and neuromuscular control over the lower body while performing special tasks in sports, the factors that change the postural control in doing these tasks can affect the performance or the demands on the muscles. The study results indicated a significant relationship between the postural control and the level of the lower limb muscles (rectus femoris and tibialis anterior) activities in the normal condition, but after fatigue, no significant relationship was observed in any lower limb muscles. Also, fatigue in the lower limb extremity muscles significantly reduced all functional test scores and the level of the muscles activities of the sportsmen. Considering the negative effect of fatigue on lower limb muscles, it can be concluded that lower limb muscles functions and their fatigue affect the lower limb performance.

The study has some limitations too. One of them is lack of a control group and randomly-chosen samples that can negatively affect the results. The other limitation of the study was lack of control over participants mental conditions, skill level, and their motivation.

With regard to the negative effects of fatigue on the function of lower body muscles, also considering that the devastating effects of fatigue is modifiable, exercises that improve and facilitate the contraction of the muscles of the lower extremity muscles and increase their ability to cope with fatigue could be used by trainers to prevent lower limb function loss caused by fatigue during prolonged exercise activities. Also for a better understanding of biomechanical differences between before fatigue phase and after that, we suggest that the kinematic, kinetic, and EMG characteristics of these two phases be compared.

\section{Acknowledgments}

This study was extracted from the master's thesis submitted to the University of Isfahan. Hereby, we appreciate the assistance of the authorities of Faculty of Physical Education of Isfahan University for allowing laboratory use, and all people participating in this research. 


\section{Conflict of Interest}

The authors declared no conflict of interests.

\section{References}

[1] Aaronson LS, Teel CS, Cassmeyer V, Neuberger GB, Pallikkathayil L, Pierce J, et al. Defining and measuring fatigue. Journal of Nursing Scholarship. 1999; 31(1):45-50. doi: 10.1111/j.1547-5069.1999.tb00420.x

[2] Allison GT, Fujiwara T. The relationship between EMG median frequency and low frequency band amplitude changes at different levels of muscle capacity. Clinical Biomechanics. 2002; 17(6):464-9. doi: 10.1016/s02680033(02)00033-5

[3] Augustsson J, Thomeé R, Karlsson J. Ability of a new hop test to determine functional deficits after anterior cruciate ligament reconstruction. Knee Surgery, Sports Traumatology, Arthroscopy. 2004; 12(5):350-6. doi: 10.1007/s00167004-0518-4

[4] Augustsson J, Thomeé R, Linden C, Folkesson M, Tranberg $\mathrm{R}$, Karlsson J. Single-leg hop testing following fatiguing exercise: Reliability and biomechanical analysis. Scandinavian Journal of Medicine \& Science in Sports. 2006; 16(2):111-20. doi: 10.1111/j.1600-0838.2005.00446.x

[5] Balogun JA, Akindele KA, Nihinlola JO, Marzouk DK. Age-related changes in balance performance. Journal of Social Work in Disability \& Rehabilitation. 1994; 16(2):5862. doi: $10.3109 / 09638289409166013$

[6] Blackburn JT, Prentice WE, Guskiewicz KM, Busby MA Balance and joint stability: The relative contributions of proprioception and muscular strength. Journal of Sport Rehabilitation. 2000; 9(4):315-28. doi: 10.1123/jsr.9.4.315

[7] Brask B, Lueke RH, Soderberg GL. Electromyographic analysis of selected muscles during the lateral step-up exercise. Physical Therapy Journal. 1984; 64(3):324-9. PMID: 6701202

[8] Brown C, Ross S, Mynark R, Guskiewicz K. Assessing functional ankle instability with joint position sense, time to stabilization, and electromyography. Journal of Sport Rehabilitation. 2004; 13(2):122-34. doi: 10.1123/jsr.13.2.122

[9] Chuter VH, de Jonge XA. Proximal and distal contributions to lower extremity injury: A review of the literature. Gait \& Posture. 2012; 36(1):7-15. doi: 10.1016/j.gaitpost.2012.02.001

[10] Cissik JM. Programming abdominal training, Part I. Strength \& Conditioning Journal. 2002; 24(1):9-15. doi: 10.1519/1533-4295(2002)024<0009:patpii>2.0.co;2

[11] Di Fabio RP. Reliability of computerized surface electromyography for determining the onset of muscle activity. Physical Therapy Journal. 1987; 67(1):43-8. PMID: 3797476

[12] Di Giulio I, Maganaris CN, Baltzopoulos V, Loram ID. The proprioceptive and agonist roles of gastrocnemius, soleus and tibialis anterior muscles in maintaining hu- man upright posture. The Journal of Physiology. 2009; 587(10):2399-416. doi: 10.1113/jphysiol.2009.168690

[13] Earl JE, Hertel J. Lower-extremity muscle activation during the star excursion balance tests. Journal of Sport Rehabilitation. 2001; 10(2):93-104. doi: 10.1123/jsr.10.2.93

[14] Escamilla RF, Fleisig GS, Zheng N, Barrentine SW, Wilk $\mathrm{KE}$, Andrews JR. Biomechanics of the knee during closed kinetic chain and open kinetic chain exercises. Medicine \& Science in Sports \& Exercise. 1998; 30(4):556-69. doi: 10.1097/00005768-199804000-00014

[15] Filipa A, Byrnes R, Paterno MV, Myer GD, Hewett TE. Neuromuscular training improves performance on the star excursion balance test in young female athletes. Journal of Orthopaedic \& Sports Physical Therapy. 2010; 40(9):551-8. doi: 10.2519/jospt.2010.3325

[16] Gehring D, Melnyk M, Gollhofer A. Gender and fatigue have influence on knee joint control strategies during landing. Clinical Biomechanics. 2009; 24(1):82-7. doi: 10.1016/j. clinbiomech.2008.07.005

[17] Gribble, P. The star excursion balance test as a measurement tool. Athletic Therapy Today. 2003; 8(2),46-47. doi: 10.1123/att.8.2.46

[18] Gribble PA, Hertel J. Effect of lower-extremity muscle fatigue on postural control. Archives of Physical Medicine \& Rehabilitation. 2004; 85(4):589-92. doi: 10.1016/j. apmr.2003.06.031

[19] Gribble PA, Hertel J, Denegar CR, Buckley WE. The effects of fatigue and chronic ankle instability on dynamic postural control. Journal of Athletic Training. 2004; 39(4):321-9. PMID: 15592604

[20] Gribble PA, Robinson RH, Hertel J, Denegar CR. The effects of gender and fatigue on dynamic postural control. Journal of Sport Rehabilitation. 2009; 18(2):240-57. doi: 10.1123/jsr.18.2.240

[21] Guskiewicz KM, Perrin DH. Research and clinical applications of assessing balance. Journal of Sport Rehabilitation. 1996; 5(1):45-63. doi: 10.1123/jsr.5.1.45

[22] Harkins KM, Mattacola CG, Uhl TL, Malone TR. Effects of 2 ankle fatigue models on the duration of postural stability. Journal of Athletic Training. 2005; 40(3):191-4. PMID: 16284640

[23] Hart JM, Fritz JM, Kerrigan DC, Saliba EN. Quadriceps inhibition after repetitive lumbar extension exercise in persons with a history of low back pain. Journal of Athletic Training. 2006; 41(3):264-9. PMCID: PMC1569566

[24] Hart JM, Fritz JM, Kerrigan DC, Saliba EN. Reduced quadriceps activation after lumbar paraspinal fatiguing exercise. Journal of Athletic Training. 2006; 41(1):79-86. PMID: 16619099

[25] Ireland ML. Anterior cruciate ligament injury in female athletes: Epidemiology. Journal of Athletic Training. 1999; 34(2):150-4. PMCID: PMC1322904

[26] Konrad P. The ABC of EMG: A practical introduction to kinesiological electromyography. Scottsdale: Noraxon U.S.A. Inc.; 2005. 
[27] Lees A. Methods of impact absorption when landing from a jump. Engineering in Medicine. 1981; 10(4):207-11. doi: 10.1243/emed_jour_1981_010_055_02

[28] Madigan ML, Pidcoe PE. Changes in landing biomechanics during a fatiguing landing activity. Journal of Electromyography \& Kinesiology. 2003; 13(5):491-8. doi: 10.1016/ s1050-6411(03)00037-3

[29] Masuda K, Masuda T, Sadoyama T, Inaki M, Katsuta S. Changes in surface EMG parameters during static and dynamic fatiguing contractions. Journal of Electromyography \& Kinesiology. 1999; 9(1):39-46. doi: 10.1016/s10506411(98)00021-2

[30] McKeon PO, Hertel J. Systematic review of postural control and lateral ankle instability, part I: Can deficits be detected with instrumented testing? Journal of Athletic Training. 2008; 43(3):293-304. doi: 10.4085/1062-605043.3.293

[31] McMullen KL, Cosby NL, Hertel J, Ingersoll CD, Hart JM. Lower extremity neuromuscular control immediately after fatiguing hip-abduction exercise. Journal of Athletic Training. 2011; 46(6):607-14. PMID: 22488185

[32] Murphy DF, Connolly DA, Beynnon BD. Risk factors for lower extremity injury: A review of the literature. British Journal of Sports Medicine. 2003; 37(1):13-29. doi: 10.1136/ bjsm.37.1.13

[33] Neptune RR, Wright IC, Van den Bogert AJ. Muscle coordination and function during cutting movements. Medicine \& Science in Sports \& Exercise. 1999; 31(2):294-302. doi: 10.1097/00005768-199902000-00014

[34] Ninos JC, Irrgang JJ, Burdett R, Weiss JR. Electromyographic analysis of the squat performed in self-selected lower extremity neutral rotation and 30 of lower extremity turn-out from the self-selected neutral position. Journal of Orthopaedic \& Sports Physical Therapy. 1997; 25(5):30715. doi: 10.2519/jospt.1997.25.5.307

[35] Neumann DA. Kinesiology of the musculoskeletal system: Foundations for rehabilitation. $3^{\text {rd }}$ ed. Philadelphia: Mosby Elsevier Health Sciences; 2013.

[36] Neumann, DA. Kinesiology of the musculoskeletal system. $1^{\text {st }}$ ed. Philadelphia: Mosby Elsevier Health Sciences; 2002.

[37] Oatis CA. Kinesiology: The mechanics and pathomechanics of human movement. Philadelphia: Lippincott Williams \& Wilkins; 2009.

[38] Orishimo KF, Kremenic IJ, Mullaney MJ, McHugh MP, Nicholas SJ. Adaptations in single-leg hop biomechanics following anterior cruciate ligament reconstruction. Knee Surgery, Sports Traumatology, Arthroscopy. 2010; 18(11):1587-93. doi: 10.1007/s00167-010-1185-2

[39] Orr R. Contribution of muscle weakness to postural instability in the elderly. European Journal of Physical \& Rehabilitation Medicine. 2010; 46(2):183-220. PMID: 20485224

[40] Ortiz A, Olson SL, Etnyre B, Trudelle-Jackson EE, Bartlett W, Venegas-Rios HL. Fatigue effects on knee joint stability during two jump tasks in women. Journal of Strength \& Conditioning Research. 2010; 24(4):1019-27. doi: 10.1519/ jsc.0b013e3181c7c5d4
[41] Paillard T, Noe F, Riviere T, Vincent M. Postural performance and strategy in the unipedal stance of soccer players at different levels of competition. Journal of Athletic Training. 2006; 41(2):172-6. PMCID: PMC1472651

[42] Parijat P, Lockhart TE. Effects of quadriceps fatigue on the biomechanics of gait and slip propensity. Gait \& Posture. 2008; 28(4):568-73. doi: 10.1016/j.gaitpost.2008.04.001

[43] Hajilou B, Anbarian M, Esmaili H, Sadeghi S. [The effect of quadriceps fatigue on electromyographic activity of some knee joint muscles during stance phase of walking (Persian)]. Journal of Sport Medicine. 2014; 6(1):73-88.

[44] Pincivero DM, Gandhi V, Timmons MK, Coelho AJ. Quadriceps femoris electromyogram during concentric, isometric and eccentric phases of fatiguing dynamic knee extensions. Journal of Biomechanics. 2006; 39(2):246-54. doi: 10.1016/j.jbiomech.2004.11.023

[45] Plisky PJ, Gorman PP, Butler RJ, Kiesel KB, Underwood FB, Elkins B. The reliability of an instrumented device for measuring components of the star excursion balance test. North American Journal of Sports Physical Therapy. 2009; 4(2):92-9. PMCID: PMC2953327

[46] Radin EL. Role of muscles in protecting athletes from injury. Acta Medica Scandinavica. 1986; 220(711):143-7. doi: 10.1111/j.0954-6820.1986.tb08943.x

[47] Riemann BL, Lephart SM. The sensorimotor system, part II: The role of proprioception in motor control and functional joint stability. Journal of Athletic Training. 2002; 37(1):80-84. PMCID: PMC164312

[48] Reiman MP, Manske RC. Functional testing in human performance. Canada: Human Kinetics; 2009.

[49] Salavati M, Moghadam M, Ebrahimi I, Arab AM. Changes in postural stability with fatigue of lower extremity frontal and sagittal plane movers. Gait \& Posture. 2007; 26(2):214-8. doi: 10.1016/j.gaitpost.2006.09.001

[50] Schaub PA, Worrell TW. EMG activity of six muscles and VMO: VL ratio determination during a maximal squat exercise. Journal of Sport Rehabilitation. 1995; 4(3):195-202. doi: $10.1123 /$ jsr.4.3.195

[51] Sidaway B, Anderson J, Danielson G, Martin L, Smith G. Effects of long-term gait training using visual cues in an individual with Parkinson disease. Journal of Physical Therapy. 2006; 86(2):186-94. PMID: 16445332

[52] Sporns O, Edelman GM. Solving Bernstein's problem: A proposal for the development of coordinated movement by selection. Child Development. 1993; 64(4):960-81. doi: $10.2307 / 1131321$

[53] Staude G, Flachenecker C, Daumer M, Wolf W. Onset detection in surface electromyographic signals: A systematic comparison of methods. EURASIP Journal on Applied Signal Processing. 2001; 2001(2):67-81. doi: 865701000191

[54] Vøllestad, N. K. Measurement of human muscle fatigue. Journal of Neuroscience Methods. 1997; 74(2):219-27. doi 10.1016/s0165-0270(97)02251-6

[55] Willson JD, Dougherty CP, Ireland ML, Davis IM. Core stability and its relationship to lower extremity function and injury. Journal of the American Academy of Orthopae- 
dic Surgeons. 2005; 13(5):316-25. doi: 10.5435/00124635200509000-00005

[56] Yaggie JA, McGregor SJ. Effects of isokinetic ankle fatigue on the maintenance of balance and postural limits. Archives of Physical Medicine and Rehabilitation. 2002; 83(2):224-8. doi: 10.1053/apmr.2002.28032

[57] Wilk KE, Escamilla RF, Fleisig GS, Barrentine SW, Andrews JR, Boyd ML. A comparison of tibiofemoral joint forces and electromyographic activity during open and closed kinetic chain exercises. The American Journal of Sports Medicine. 1996; 24(4):518-27. doi: $10.1177 / 036354659602400418$

[58] Winter DA. Biomechanics and motor control of human movement. $4^{\text {th }}$ ed. New Jersey: Wiley Blackwell; 2009.

[59] Winter DA. Biomechanics of normal and pathological gait: Implications for understanding human locomotor control. Journal of Motor Behavior. 1989; 21(4):337-55. doi: 10.1080/00222895.1989.10735488 
- 